\title{
OSCILLATORY SOLUTIONS FOR CERTAIN DELAY-DIFFERENTIAL EQUATIONS
}

\author{
GEORGE SEIFERT
}

(Communicated by George R. Sell)

\begin{abstract}
The existence of oscillatory solutions for a certain class of scalar first order delay-differential equations is proved. An application to a delay logistic equation arising in certain models for population variation of a single specie in a constant environment with limited resources for growth is considered.
\end{abstract}

It is known (cf. $[\mathbf{1}, \mathbf{2}])$ that all solutions of the delay logistic equations

$$
N^{\prime}(t)=N(t)(a-b N(t)-N(t-1)), \quad t>0,
$$

with $N(t)=N_{0}(t)>0,-1 \leq t \leq 0, N_{0}$ continuous, and $a$ and $b$ positive constants, satisfy $N(t) \rightarrow a /(b+1)$ as $t \rightarrow \infty$ whenever $b>1$. In [3] it was shown that for any $b>0$, there exists $a(b)>0$ and that if $0<a<a(b)$, there exist solutions $N(t)$ of (1) which do not oscillate about the equilibrium $N=a /(b+1)$; in particular, such that, $N(t)>a /(b+1)$ for $t \geq 0$. It is the purpose of this paper to show that for this same $a(b)$, if $a>a(b)$, there exist oscillatory solutions about this equilibrium solution. In case $b<1$, this is known; in fact, a Hopf bifurcation (cf. [1]) shows the existence for certain $a$ of nonconstant positive periodic solutions. However, if $b>1$, the fact that some solutions of (1) approach $a /(b+1)$ in an oscillatory fashion seems to be new.

The above mentioned result for (1) will follow from a result for a more general scalar delay-differential equation of the form

$$
y^{\prime}(t)=L\left(y_{t}\right)+N\left(t, y_{t}\right), \quad t>0 .
$$

Here $y_{t}=y(t+\theta),-1 \leq \theta \leq 0$, and we assume

$\left(\mathrm{H}_{1}\right) L(\phi)$ is continuous and linear on $C=C([-1,0], R)$ and $N(t, \phi)$ is continuous on $R \times C$ and satisfies

$$
|N(t, \phi)| \leq M(t)\|\phi\|^{2}, \quad \phi \in C,\|\phi\| \leq B_{0}, t \geq 0 ;
$$

where the norm in $C$ is defined by $\|\phi\|=\sup \{|\phi(\theta)|:-1 \leq \theta \leq 0\}$, and $\int_{0}^{\infty} M(t) d t$ $<\infty$;

$\left(\mathrm{H}_{2}\right)$ The characteristic equation for

$$
y^{\prime}(t)=L\left(y_{t}\right)
$$

has a pair of simple pure imaginary roots $\pm i \beta, \beta>0$, and all other roots have negative real parts.

Received by the editors October 16, 1987.

1980 Mathematics Subject Classification (1985 Revision). Primary 34K20.

Key words and phrases. Delay-differential equations, oscillatory solutions, delay-logistic equation. 
REMARK 1. Under assumption $\left(\mathrm{H}_{2}\right)$, there exists a nonconstant periodic solution $y^{*}(t)$ of (3) and positive numbers $\rho^{*}$ and $B, \rho^{*}<1, B<B_{0} / 2$, such that

$$
\begin{gathered}
\max \left\{y^{*}(t): t \in R\right\} \geq \rho^{*}, \min \left\{y^{*}(t): t \in R\right\} \leq-\rho^{*}, \\
\left|y^{*}(t)\right| \leq B, \quad t \in R .
\end{gathered}
$$

This follows from standard theory for solutions of (3); cf., for example, Hale's monograph [4].

DEFINITION. The real-valued function $f(t)$ on $[0, \infty)$ is oscillatory if there exist $t_{n} \rightarrow \infty$ as $n \rightarrow \infty, t_{n+1}>t_{n}$, such that $(-1)^{n} f\left(t_{n}\right)>0, n=1,2, \ldots$

REMARK 2. If $f(t)$ is continuous and oscillatory in this sense, clearly $f$ must have an unbounded sequence of zeros and cannot be identically zero on any half infinite interval $\left[t_{0}, \infty\right), t_{0} \geq 0$.

THEOREM 1. If $\left(\mathrm{H}_{1}\right)$ and $\left(\mathrm{H}_{2}\right)$ hold, there exists $\delta_{0}>0$ such that for each $\delta$, $0<\delta<\delta_{0}$, (2) has an oscillatory solution $y=w(t)$ such that $|w(t)| \leq \delta, t \geq 0$.

PROOF. Let $u(t)$ be the fundamental solution for (3); i.e., let $u(t)$ solve (cf. appendix)

$$
\begin{aligned}
& u^{\prime}(t)=L\left(u_{t}\right), \quad t>0, \\
& u(0)=1, \\
& u(t)=0, \quad-1 \leq t<0 .
\end{aligned}
$$

From $\left(\mathrm{H}_{2}\right)$ it follows that there exists $K>0$ such that $|u(t)| \leq K, t \geq 0$; again cf. [4, Chapter 7]. For this $K$, and $\rho^{*}$ and $B$ as in Remark 1, fix $\varepsilon>0$ such that

$$
\varepsilon \int_{0}^{\infty} M(t) d t \leq \frac{\rho^{*}}{8 B^{2} K}<(4 B K)^{-1}
$$

note that $\rho^{*} \leq B$.

Let $X(B)$ denote the set of real functions $z$ continuous on $[-1, \infty)$ such that $z(t)=y^{*}(t),-1 \leq t \leq 0$, where $y^{*}(t)$ is the periodic solution of $(3)$ as described in Remark 1 , and $|z(t)| \leq 2 B, t \geq 0$. With the topology of uniform convergence on compact subsets of $[-1, \infty)$, the set $X$ of all real functions continuous on $[-1, \infty)$ is a locally convex linear topological space over the reals, and clearly $X(B) \subset X$. Define the map $T$ on $X(B)$ to $X$ by

$$
\begin{aligned}
(T z)(t) & =y^{*}(t)+\frac{1}{\varepsilon} \int_{0}^{t} u(t-s) N\left(s, \varepsilon z_{s}\right) d s, \quad t>0 \\
& =y^{*}(t), \quad-1 \leq t \leq 0
\end{aligned}
$$

for any $z \in X(B)$.

Using (5) with $\left(\mathrm{H}_{1}\right)$ and the boundedness property of $u(t)$, we have

$$
(T z)(t) \leq B+4 K B^{2} \varepsilon \int_{0}^{t} M(s) d s \leq 2 B, \quad t \geq 0
$$

therefore $T z \in X(B)$.

Using (6) and the properties of $u(t)$ given in (4) it follows that

$$
\begin{aligned}
\frac{d}{d t}(T z)(t) & =y^{* \prime}(t)+\frac{1}{\varepsilon} N(t, \varepsilon z(t))+\frac{1}{\varepsilon} \int_{0}^{t} u^{\prime}(t-s) N(s, \varepsilon z(s)) d s \\
& =y^{* \prime}(t)+\frac{1}{\varepsilon} N(t, \varepsilon z(t))+\frac{1}{\varepsilon} \int_{0}^{t} L\left(u_{t-s}\right) N(s, \varepsilon z(s)) d s
\end{aligned}
$$


So since $L$ is bounded, $z \in X(B), u(t)$ is bounded for $t>0$, and $N$ satisfies the conditions in $\left(\mathrm{H}_{1}\right)$, it follows that there exists a constant $C(\varepsilon)$ and that

$$
\frac{d}{d t}(T z)(t) \leq C(\varepsilon), \quad t>0
$$

By a standard argument using the Ascoli-Arzela theorem, it then follows that $T X(B)$ is precompact in the topology of $X$, and by the Schauder-Tychonov fixed point theorem, there exists a $z^{*} \in X(B)$ such that

$$
\begin{gathered}
z^{*}(t)=y^{*}(t)+\frac{1}{\varepsilon} \int_{0}^{t} u(t-s) N\left(s, \varepsilon z^{*}(s)\right) d s, \quad t>0, \\
z^{*}(t)=y^{*}(t), \quad-1 \leq t \leq 0 .
\end{gathered}
$$

Since $u(t)$ is a fundamental solution for (3), it follows that $z^{*}(t)$ solves

$$
\begin{gathered}
z^{\prime}(t)=L\left(z_{t}\right)+\frac{1}{\varepsilon} N\left(t, \varepsilon z_{t}\right), \quad t>0, \\
z(t)=y^{*}(t), \quad-1 \leq t \leq 0,
\end{gathered}
$$

and so $y(t)=\varepsilon z^{*}(t)$ solves (2) for $t>0$ with $y(t)=\varepsilon y^{*}(t)$ for $-1 \leq t \leq 0$.

If

$$
R_{0}(t)=\frac{1}{\varepsilon} \int_{0}^{t} u(t-s) N\left(s, \varepsilon z^{*}(s)\right) d s, \quad t \geq 0,
$$

then $z^{*}(t)=y^{*}(t)+R_{0}(t), t \geq 0$, and using the properties of $u$ and $N$ and the fact that $z^{*} \in X(B)$ it follows that

$$
\left|R_{0}(t)\right| \leq 4 B^{2} K \varepsilon \int_{0}^{t} M(s) d s \leq \frac{\rho^{*}}{2}, \quad t \geq 0 .
$$

So

$$
\left|z^{*}(t)-y^{*}(t)\right| \leq \rho^{*} / 2, \quad t \geq 0 .
$$

But using the properties of $y^{*}(t)$ mentioned in Remark 1, there exists $t_{n} \rightarrow \infty$ as $n \rightarrow \infty, t_{n+1}>t_{n}$, such that $y^{*}\left(t_{n}\right) \geq \rho^{*}, n=1,2, \ldots$ Using (8) it follows easily that

$$
z^{*}\left(t_{n}\right) \geq \rho^{*} / 2, \quad n=1,2, \ldots
$$

Similarly, there exists a sequence $\tau_{n} \rightarrow \infty$ as $n \rightarrow \infty, \tau_{n+1}>\tau_{n}$, such that $y^{*}\left(\tau_{n}\right) \leq-\rho^{*}$, and so

$$
z^{*}\left(\tau_{n}\right) \leq-\rho^{*} / 2, \quad n=1,2, \ldots
$$

Thus the solution $y(t)=\varepsilon z^{*}(t) \equiv w(t)$ of (2) is oscillatory. Now define $\varepsilon_{0}$ to be the supremum of the set of all $\varepsilon>0$ for which this argument holds. Since for such $\varepsilon>0,|w(t)| \leq \varepsilon B$, with $B$ as in Remark 1 , and if we take $\delta_{0}=2 \varepsilon_{0} B$, our theorem is proved. Note that from (5), $\varepsilon_{0} \leq(4 B K M)^{-1}$, where $M=\int_{0}^{\infty} M(t) d t$.

REMARK 3. If $\beta$ is as in $\left(\mathrm{H}_{2}\right)$, it can be shown that the $t_{n}$ and $\tau_{n}$ in our proof above can be chosen such that

$$
t_{n+1}-t_{n} \leq 2 \pi / \beta, \text { and } \tau_{n+1}-\tau_{n} \leq 2 \pi / \beta .
$$

This follows because $y^{*}(t)$ can be chosen to be a linear combination of $\sin \beta t$ and $\cos \beta t$. We omit the details. 
We now return to the delay logistic equation (1) with $b>1$. If we make the change of variables $x(t)=N(t)-a /(b+1)$ (1) becomes

$$
x^{\prime}(t)=-(a /(b+1)+x(t))(b x(t)+x(t-1)),
$$

and the linear part of $(9)$ is the equation

$$
x^{\prime}(t)=-(a /(b+1))(b x(t)+x(t-1)) .
$$

It is not difficult to see that all roots of the characteristic equation for (10) have negative real part, cf. [5]. From a result in [3], it also follows that if

$$
a>(b+1) / m(b)
$$

where $m(b)$ is the unique root of $b=m(\log m-1)$, then all roots of this characteristic equation are nonreal. A direct examination of this characteristic equation also shows that all nonreal roots must be simple.

Under the change of variable $y(t)=x(t) \exp (\mu t)$, where $\mu$ is a real constant, (9) becomes

$$
y^{\prime}(t)=A(\mu) y(t)+B(\mu) y(t-1)+f(y(t), y(t-1)) \exp (-\mu t)
$$

where $A(\mu)=\mu-a b /(b+1), B(\mu)=-a e^{\mu} /(b+1)$, and

$$
f(y, z)=-\left(b y^{2}+y z e^{\mu}\right) .
$$

It is easy to see that if $\alpha$ is the real part of a root of the characteristic equation for (9), then $\mu+\alpha$ is the real part of a corresponding root of the characteristic equation for the linear part of (12), namely

$$
y^{\prime}(t)=A(\mu) y(t)+B(\mu) y(t-1) .
$$

So if we choose $\mu=-\max \{\operatorname{Re} \lambda: \lambda$ is a root of the characteristic equation for $(10)\}$, then the characteristic equation for (13) has pure imaginary roots $\pm i \beta, \beta>0$, which are simple if (11) holds. Also all other roots of this equation for (13) have negative real parts. Clearly $\mu>0$. So we see that all the hypotheses of Theorem 1 are satisfied for (11) and we have the following.

THEOREM 2. If $b>1$ and $a>(b+1) / m(b)$, where $m(b)$ is as defined above, then there exist oscillatory solutions of (9) of arbitrarily small amplitude; i.e., there exist solutions of (1) which oscillate about $a /(b+1)$.

The proof of this theorem now follows easily, since by Theorem 1, there exist such oscillatory solutions $y(t)$ of $(12)$ and so the corresponding solutions $x(t)=$ $y(t) \exp (-\mu t)$ are also oscillatory.

An open question presents itself: under the hypotheses of Theorem 2, are all solutions of (9) oscillatory?

A PPENDIX. In the strict sense, the initial function on $[-1,0]$ for the equation defining $u(t)$ in (4) is not in $C$. What is really involved here (a point not entirely clear in [4]) is that $u(t)$ solves the initial value problem

$$
\begin{array}{rlrl}
u^{\prime}(t) & =\int_{-t}^{0} u(t+s) d \eta(s), & & 0 \leq t<1, \\
& =\int_{-1}^{0} u(t+s) d \eta(s), & & t \geq 1, \\
u(0) & =1
\end{array}
$$


where $\eta(s)$ is a function of bounded variation which by the Riesz representation theorem characterizes $L$; i.e. is such that $L(\phi)=\int_{-1}^{0} \phi(s) d \eta(s)$ for $\phi \in C$. This initial value problem can be shown to have a solution in a fairly standard way such as by the method of successive approximations.

\section{REFERENCES}

1. G. Seifert, On a delay-differential equation for single specie population variations, Nonlinear Analysis, Theory Methods \& Appl. 11 (1987), 1051-1059.

2. S. M. Lenhart and C. C. Travis, Global stability of a biological model with time delay, Proc. Amer. Math. Soc. 96 (1986), 75-78.

3. G. Seifert, Oscillation of solutions of a population equation with delay, Nonlinear Analysis and Applications, Lecture Notes in Pure and Appl. Math., 109, V. Lakshmikantham, ed., 1987, Marcel Dekker, pp. 549-554.

4. J. K. Hale, Theory of functional differential equations, Springer-Verlag, New York, 1977.

5. R. Bellman and K. Cooke, Differential difference equations, Academic Press, New York, 1963.

Department of Mathematics, Iowa State University, Ames, Iowa 50011 